\title{
Gaussian Noise Filtering Techniques using New Median Filter
}

\author{
H S Shukla \\ Deen Dayal Upadhyay \\ Gorakhpur \\ University,Gorakhpur (India)
}

\author{
Narendra Kumar \\ Deen Dayal Upadhyay \\ Gorakhpur \\ University, Gorakhpur (India)
}

\author{
R P Tripathi \\ Graphic era university \\ Dehradun (India)
}

\begin{abstract}
Image filtering is a essential part of image processing. There are various filter are available but gives better result only for particular noise. Median filter are one of them median filter give better result for 'salt and pepper' noise but when we use this filter in Gaussian noise not give better result. We are proposing a new median filter with some modification of existing median filter pixel values for Gaussian noise. We are also showing comparison with existing various size of median filtering also showing the result using some noise parameters.
\end{abstract}

\section{Keywords}

Median filter, Gaussian noise, Peak Signal to Noise Ratio, Maximum Absolute Error, impulse noise.

\section{INTRODUCTION}

There are various denoising methods that have been proposed for example neighborhood filtering, total variation minimization, Wiener filtering, Gaussian scalar mixture, methods based on partial differential equation which is given by Thaipanich and Kuo [5] Adaptive low pass filtering, adaptive median filtering and geometric filter. Gaussian filtering have some important applications. However, they have the disadvantage of blurring the edges due to non similar patterns averaging are discussed by Manjón et al [7]. Image quality is essential for many applications But, degradation of images due to different kinds of noises is highly probable. Many edge preserving filters have been proposed in order to avoid this problem. The Anisotropic Diffusion Filter (ADF) is also popular due to Perona and Malik [8] Manjon, Robles and Thacker [9] Gerig et al [10] respectively. ADF respects edges by averaging pixels in the orthogonal direction of the local gradient,to estimate only the signal value in the central pixel of the window. An inverse transform is used and then wavelet based filters have also been applied to image denoising given by Wood and Johnson [11]. Some wavelet based techniques given by established the correlation of wavelet coefficients between consecutive scales to distinguish noise from meaningful data. In this paper, we propose an effective noise reduction technique for the image.

\subsection{Noise Model}

Image noise arise either at the time of image generation (e.g. when we use camera and photographic films to capture an image) or at the time of image transmission. Different types of noise having certain characteristics likes their intensity. The film grain noise does not has statistical correlation for distance between samples greater than the grain size. Therefore film grain noise is a white noise in two dimensional random process. If its level is low it can be treated as a poison distributed noise otherwise it can be treated as a Gaussian distributed noise. Thus it can be said as signal dependent noise. Another type of noise that is present during the image transmission is Salt-Pepper noise [3].

\subsection{Gaussian Noise Model}

There are various types of noise arise during the image transmission [1] and /or acquisition Gaussian noise is one of them. Feature of Gaussian noise is that it evenly distributed over the signal. It means noisy image pixel is the sum of the actual pixel value and a random Gaussian distributed noise value. This type of noise has a Gaussian distribution, the distribution function given by,

Where:

$$
\mathrm{p}(\mathrm{z})=\frac{1}{\sqrt{2 \pi} \sigma} e^{-\frac{(\mathrm{z}-\mu)^{2}}{2 \sigma^{2}}}
$$

$\mathrm{z}=$ gray level.

$\mu=$ mean or average of function

$\sigma=$ standard deviation of noise

$\mathrm{p}(\mathrm{z})=$ probability density function

Default value into image, Gaussian noise with zero mean and variance is 0.05

\section{FILTERING}

Elimination of noise is done by image processing, as noise leads to the error in the image. Presence of noise causes disturbs the information present in the image. These noise information can be introduced because of so many reasons like: acquisition process due to cameras quality and restoration, acquisition condition, such as illumination level, calibration and positioning or it can be a function of the scene environment. Noise elimination is a part of image processing. A digital filter [1][3] is used to remove noise from the degraded image. As any noise in the image can be result in serious errors. Noise is an unwanted signal, which is manifested by undesirable information. Thus the image, which gets contaminated by the noise, is the degraded image and using different filters that is used to filter this noise and also used for image enhancement i.e. linear filters or nonlinear filters [4].The design-of digital filters involves three basic steps: (i) the specification of the desired properties of the system,(ii) the approximation of these specifications using a causal discrete time system, and (iii) the realization of the system using finite precision arithmetic[2].

\subsection{Median Filter}

A median filter is a nonlinear filters unlike the mean filter. The median filter follows moving window principle that is same as the mean filter. A $3 \times 3,5 \times 5$, or $7 \times 7$ kernel of pixels is scanned over pixel matrix of the entire image. The median pixel values in the window is evaluated, and the center pixel of the window is replaced with the evaluated median. duiring Median filtering, first sorting all the pixel values from the surrounding neighborhood into numerical order and then replacing the pixel being considered with the middle pixel value. Note that the median value must be written to a separate array or buffer so that the results are not corrupted as the process is performed. 
Centered at i, $\mathrm{j}$ as $N_{i j}$ the filter can be described by: $y_{i j}=\operatorname{med}\left(\left\{x_{u v} \mid x_{u v} \in N_{i j}\right\}\right)$

\subsection{Multi-stage Median Filters}

Median filters preserve straight edges, but not good at sharp corners . This difficulty is usually dealt with by forming a multistage median filter; this filter responds with the median of a set of different medians, obtained in new neighborhoods

$y_{i j=m e d}(z 1, z 2, z 3, z 4)$ $z 1=$ $\operatorname{med}\left(\left\{x_{u v} \mid x_{u v} \in N_{i j}^{1} \quad\right\}\right)$

$z 2=\operatorname{med}\left(\left\{x_{u v} \mid x_{u v} \in N_{i j}^{2}\right\}\right)$

$z 3=\operatorname{med}\left(\left\{x_{u v} \mid x_{u v} \in N_{i j}^{3} \quad\right\}\right)$

$z 4=\operatorname{med}\left(\left\{x_{u v} \mid x_{u v} \in N_{i j}^{4} \quad\right\}\right)$

where $N^{1}$ is a vertically extended neighbourhood, $N^{2}$ is a horizontally extended neighborhood, and $N^{3}$ and $N^{4}$ are diagonal neighborhoods.

\section{PROBLEM FORMULATION AND SOLUTION}

Noise is of many types. Thus image noise can be Gaussian, Uniform or impulsive distribution. Impulse noise generates pixels with gray level values not consistent with their local neighborhood values. The impulse noise appears as sprinkle of light or dark spots in the image. This impulse noise can be eliminated or the degraded image can be enhanced by the use of advance filter. Due to certain disadvantages of linear filters, nonlinear method of filtering has been proposed in this paper. The filter cannot be modeled by convolutions fall into the category of nonlinear filter. Nonlinear filter can be very effective in removing the impulse noise. The median filter replaces the middle pixel value with the median value of the window. The most popular non-linear filter is the median filter. When considering a small neighborhood it is highly efficient and has proved to be very effective in removing noise of an impulsive nature despite its simple definition. Nevertheless, the median filter often fails to provide sufficient smoothing of non-impulsive noise and its result is sometimes unpredictable. Several techniques have been proposed which try to take the advantage of the average performance of the median filter, either to evaluate noise density, set up parameters or to guide the filtering process.

\subsection{Solution Methology}

In any noise removal technique it is required to keep original property of image .We are providing some modification in pixel position of noisy image and input it into median filter. the output image are improved due to this techniques.

\subsection{Mathematical Analysis}

The performance of the proposed filters for removal of impulse noise and to evaluate their comparative performance are defined as follows:

Mean Squared Error (MSE):

$$
\frac{1}{w \cdot h} \sum_{i} \sum_{j}\left(x_{i, j}-\hat{x}_{i, j}\right)^{2}
$$

Peak Signal to Noise Ratio $=20 \log _{10} \frac{2^{B}-1}{\sqrt{\mathrm{MSE}}}$

Maximum Absolute Error $(\mathrm{MAE})=\max _{i, j}\left|x_{i, j}-\hat{x}_{i, j}\right|$

Here $w$ and $h$ denote the image width and height, respectively; $x_{i, j}$ and $\hat{x}_{i, j}$ denote the original and reconstructed pixel value in the $i^{\text {th }}$ row and $j^{\text {th }}$ column of the image; and $B$ denotes the dynamic range (in bits) of the original image.Technically speaking, PSNR is a quality metric (because increasing PSNR values indicates increasing reconstructed image fidelity) while MSE and MAE are distortion metrics.

\section{PROPOSED WORK}

The proposed median technique is the modification of median process during filtering with horizontal and vertical neighborhood pixel values. Proposed modified median algorithm is:

\section{Algorithm}

Step 1: take corrupted input image of size $\mathrm{m} \times \mathrm{n}$

Step 2: convert image into double type assign it into variable $\mathrm{A}$ A=double(corrupted image)

Step 3:.take image into $\mathrm{m} x \mathrm{n}$ matrix $\mathrm{M}=[\mathrm{m} \times \mathrm{n}]$

Step 4: change the pixel position of corrupted image and put it into median filter

$$
\begin{aligned}
& \text { for } i=2 \text { to } m-1 \\
& \text { for } j=2 \text { to } n-1 \\
& M(1)=A(i-1, j-1) \\
& M(2)=A(i-1, j) \\
& M(3)=A(i-1, j+1) \\
& M(4)=A(i, j-1) \\
& M(5)=A(i, j) \\
& M(6)=A(i, j+1) \\
& M(7)=A(i+1, j-1) \\
& M(8)=A(i+1, j) \\
& M(9)=A(i+1, j+1) \\
& A(i, j)=m e d i a n(M)
\end{aligned}
$$

end

Step 5: output image A

Step 6:Stop

\section{RESULT}

Experimental result has been given in fig 1 and fig 2 for a image a1.png Result of 3 x 3 Median filter and 7 x 7 Median Filter.
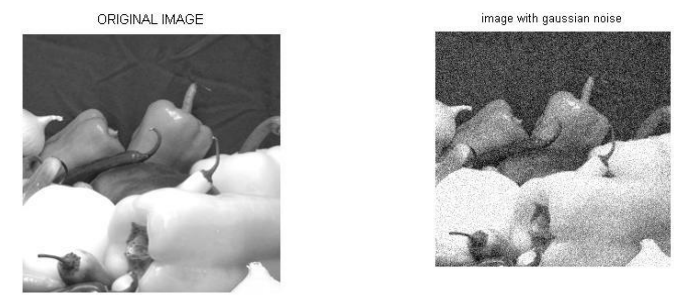

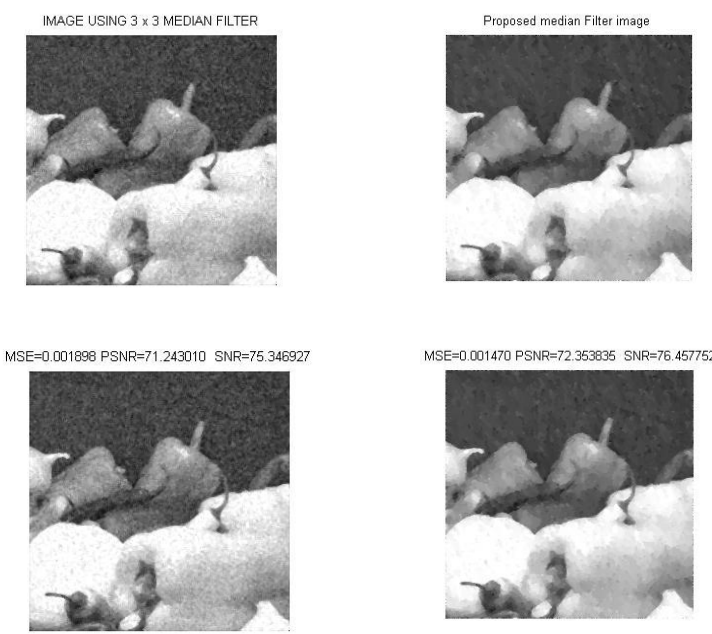

Fig1: result with image a1.png 256 x 256 using $3 \times 3$ median filter and proposed median filter $7 \times 7$ median filter
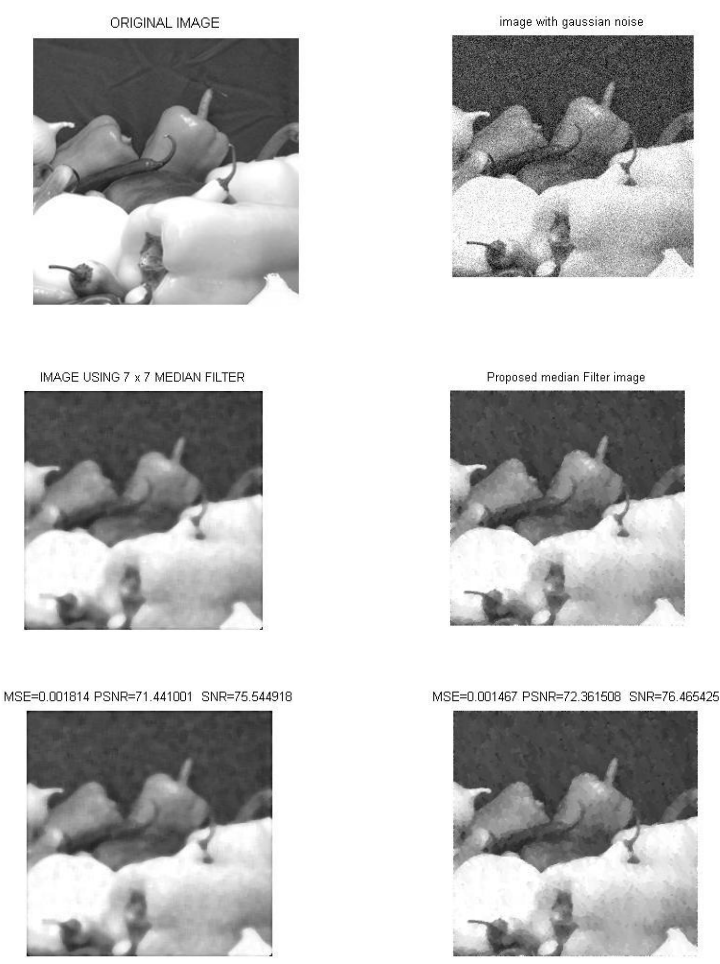

Fig2: Result with image a1.png 256 x 256 using 3x 3 median filter and proposed median filter

Variations of MSE ,PSNR and SNR of the noisy image given on table using $3 \times 3$ median filter and $7 \times 7$ median filter. The proposed technique is little better than existing median filtering.

Following tables showing the improved MSE,SNR and PSNR
Table1: performance evaluation using $3 \times 3$ median filter and proposed filter

\begin{tabular}{|l|l|l|}
\hline Values matrices & $\begin{array}{l}\mathbf{3} \times \mathbf{3} \text { median } \\
\text { filter }\end{array}$ & Proposed filter \\
\hline MSE & 0.001470 & 0.001898 \\
\hline SNR & 71.243010 & 72.353835 \\
\hline PSNR & 75.346927 & 75.457752 \\
\hline
\end{tabular}

Table 2: performance evaluation using 7 x 7 median filter and proposed filter

\begin{tabular}{|l|l|l|}
\hline matrices & $\begin{array}{l}\mathbf{7} \text { x 7 median } \\
\text { filter }\end{array}$ & Proposed filter \\
\hline MSE & 0.001814 & 0.001467 \\
\hline SNR & 71.441001 & 72.361508 \\
\hline PSNR & 75.544918 & 76.465425 \\
\hline
\end{tabular}

\section{CONCLUSION}

This paper proposed new non-linear median filters to remove the impulse noise from the images. All the filtering techniques have been implemented in MATLAB 7.1 with Pentium-core2due processor. The schemes are implemented using images a1.png. The impure contaminations include Gaussian noise. i.e. better restored results and other parameter for restoration compared to the existing schemes when Gaussian noise is considered. There is another emerging area of image restoration technique, called Blind De-Convolution technique. In this direction further work can be extended by bio based optimization techniques.

\section{ACKNOWLEDGMENTS}

We express our sincere thanks to Mr Ravi Verma of CSE Department, for his guidance and tremendous support. His cooperation and suggestions have made the timely completion of our project possible.

Also we would like to thank our project guide $\operatorname{Dr} R \mathrm{P}$ Tripathi for their support, cooperation and suggestions.

\section{REFERENCES}

[1] A.K.Jain, "Fundamentals of Digital Image Processing", Engelwood Cliff, N. J.: Print ice Hall, 2006.

[2] S. K. Satpathy, S. Panda, K. K. Nagwanshi and C. Ardil, "Image Restoration in Non-Linear Filtering Domain using MDB approach", International Journal of Information and Communication Engineering, Vol. 6, No. 1, 2010.

[3] J.S.Lee,"Digital Image Enhancement and Noise Filtering by use of Local Statistics", IEEE Trans. On Pattern Analysis and. Machine Intelligence, Vol.PAMI-29, March,1980.

[4] H.Taub, D.L. Schilling, "Principles of Communication Systems", TMH,1991.

[5] Tanaphol Thaipanich and Jay Kuo, "An Adaptive Nonlocal Means Scheme for Medical Image Denoising", In Proceedings of SPIE Medical Imaging, Vol. 7623, San Diego, CA, USA, February 2010

[6] Su Cheol Kang and Seung Hong Hong, "A Speckle Reduction Filter using Wavelet- Based Methods for 
Medical Imaging Application", In Proceedings of 23rd Annual International Conference of the IEEE Engineering in Medicine and Biology Society, Vol. 3, pp. 2480-2483,Istanbul, Turkey, October 2001

[7] Jose V. Manjón, Neil A. Thacker, Juan J. Lull , Gracian Garcia-Marti , Luis Marti-Bonmati and Montserrat Robles, "Multicomponent MR Image Denoising", Journal of Biomedical Imaging, Vol. 2009, No. 18, pp. 127,2009

[8] Perona and Malik, "Scale-space and edge detection using anisotropic diffusion", IEEE Transaction on Pattern Analysis and Machine Intelligence, Vol. 12, No. 7, pp. 629-639, 1990
[9] Manjon, Robles and Thacker, "Multispectral MRI denoising using non-local means", In Proceedings of MIUA, pp. 41-46, Aberystwyth, 2007

[10] Gerig, Kubler, Kikinis and Jolesz, "Nonlinear Anisotropic Filtering of MRI Data", IEEE Transaction on Medical Imaging, Vol. 11, No. 1, pp. 221-232, 1992

[11] Wood and Johnson, "Wavelet Packet Denoising of Magnetic Resonance Images: Importance of Rician Noise at Low SNR", Magnetic Resonance in Medicine, Vol. 41, No. 1, pp. 631-635, 1999 